\title{
Le(s) lieu(x) du hip-hop au Brésil
}

Sofiane Ailane

\section{CpenEdition}

\section{Journals}

Édition électronique

URL : http://journals.openedition.org/pa/156

DOI : $10.4000 /$ pa. 156

ISSN : 2273-0362

\section{Éditeur}

Université Lumière Lyon 2

\section{Édition imprimée}

Date de publication : 31 octobre 2012

Pagination : 204-219

ISBN : 1634-7706

ISSN : 1634-7706

Référence électronique

Sofiane Ailane, « Le(s) lieu(x) du hip-hop au Brésil », Parcours anthropologiques [En ligne], 8 | 2012, mis en ligne le 20 avril 2013, consulté le 01 mai 2019. URL : http://journals.openedition.org/pa/156 ; DOI : 10.4000/pa.156 


\section{Le(s) lieu(x) du hip-hop au Brésil}

Sofiane Ailane

Université Lumière Lyon 2, CREA

Le hip-hop réunit quatre expressions d'origines diverses. le rap, tout d'abord, est une forme de "parler-chanter » exécuté sur des rythmes de base produits par le Disc Jockey (DJ). Le Dj'ing, cette capacité à créer ou bien réinventer des sons, est une composante importante du hip-hop, puisqu'avec la volubilité du rappeur, elle est ce qui caractérise la musique hip-hop, c'est à dire la nécessaire maîtrise des outils de productions musicales (platines, samplers, ordinateurs, logiciels de traitement etc.). Le breakdance, ensuite, est l'expression corporelle du hip-hop. C'est une danse à dimension athlétique dont l'esthétique se base sur la rupture de flux dans des mouvements amples et fluides (Rose, 1998). Le graffiti, enfin, correspond à l'art plastique hip-hop. Il se donne à voir généralement sur le mobilier urbain (murs, métros, immeubles par exemple) au travers de fresques colorées. Bien souvent le graffiti reprend le pseudonyme de l'auteur. Il se distingue par l'usage des techniques de spray et par la superposition des couleurs (Bazin, 1998).

Au cours de mes recherches doctorales, je me suis intéressé aux pratiques du hip-hop dans un contexte urbain : la ville de Fortaleza' au Brésil. Dans la

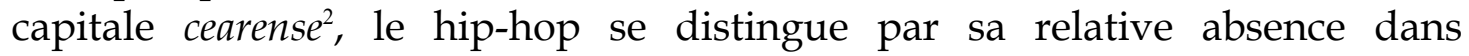
l'ambiance sonore. Cependant mes "déambulations» dans la ville $\mathrm{m}$ 'ont permis de faire connaissance avec le hip-hop tel qu'il se pratique à Fortaleza. J'ai pu alors me rendre compte que les éléments du hip-hop étaient fortement présents dans les activités de plusieurs associations et d'Organisations Non Gouvernementales qui se revendiquent comme faisant partie du « hip-hop organizado », le « hip-hop organisé ». Ces institutions jouent, entre autres, un rôle de représentant de quartier et tentent de conduire un travail social auprès des « jeunes » de la ville avec pour objectif de donner à voir « le jeune habitant de la periferia »d'une manière plus positive.

${ }^{1}$ Fortaleza, capitale de l'Etat du Ceará est du point de vue démographique la cinquième ville du Brésil avec ses 2,5 millions d'habitants. Fortaleza et sa région métropolitaine constituent aujourd'hui un pôle attractif pour les populations de l'Intérieur de l'Etat mais aussi de régions voisines, désireuses de vivre dans des meilleures conditions. Ce n'est pas un phénomène récent puisque la population de Fortaleza s'est formée à partir d'un exode rural des plus massifs tout au long du XIXe siècle et XXe siècle. (Silva, 2000)

${ }^{2}$ Gentilé des habitants de l'État du Ceará. 
Il me semble important de revenir sur le terme de periferia afin de mieux saisir l'environnement social dans lequel travaillent les militants du « hip-hop organisé ». Comme la notion de «banlieue » dans le contexte français, celle de periferia au Brésil est difficile à définir et à saisir (Kokoreff, 2003). C'est un espace qui se donnerait à voir homogène, tout au moins homogène dans les rangs que forme sa population, mais aussi par le type de vie qu'elle mène. La favela apparait comme l'idéal type de la periferia; elle formerait une ville enclavée avec ses propres lois, ses codes, ses pratiques religieuses mystiques et un quotidien différent du reste de la ville (Valladares, 2000). Cette vision de la periferia conduit à la penser comme un espace uniforme et même unitaire.

Les habitants de la periferia sont également pris dans une imagerie négative, affublés de surnoms qui résument un sentiment de rejet envers eux. Ils seraient des «marginais, vagabundos, sem vergonha, sem futuro, maconheiros, macumbeiros... $»^{3}$. Le terme même de favelado qui définit l'habitant de la favela est devenu au fil du temps une insulte. Les habitants de la periferia sont ainsi marqués par des attributs négatifs imaginés comme leurs caractéristiques propres (Paz Tella, 2008). La periferia serait donc, par essence, l'espace de la pauvreté, de la violence et de l'anomie. Dépeinte comme le lieu des anomalies sociales, elle possède une aura sulfureuse qui envahirait par les épisodes de violences, des espaces plus nobles de la ville, comme le centro ou encore le front de mer.

À ce titre, habiter la periferia, ce serait habiter une "autre-ville», car bien plus que ce qui est à la périphérie d'un centre, le terme désigne aussi ce qui lui est opposé, dans une dichotomie riche/pauvre, inclus/exclu, dominant/dominé que l'on retrouve aussi dans les discours politiques et les médias. La periferia reviendrait à désigner l'espace qui se situe loin du centre, mais aussi loin des modes de vie de ce dernier. Elle devient par cette forte composante symbolique, avant toute chose un espace de la marginalité.

Néanmoins, la réalité du terrain montre que la periferia n'est pas l'espace homogène que l'on voit dans les journaux ou les reportages télévisés. La periferia est un espace hétérogène et protéiforme à plusieurs niveaux. Il serait bien simpliste de la réduire à des zones d'extrême précarité. En effet, il existe aussi dans ce qu'on appelle la periferia des quartiers résidentiels habités par des classes moyennes ou bien des zones de commerce intense. À Fortaleza, des quartiers comme ceux de Conjunto Ceará ou de Messejana, par exemple, sont des zones qui présentent toutes les commodités existantes dans le centreville, y compris de nombreux établissements commerciaux et des banques, malgré leurs positions périphériques. Différemment des clichés, la periferia n'est donc pas uniquement le lieu d'habitation des plus pauvres et misérables,

${ }^{3}$ Je cite quelques exemples que j'ai pu tirer de mes conversations avec des Fortalezences qui mettaient en avant leurs bonnes mœurs en opposition à la morale douteuse des habitants pauvres de la ville qui seraient «marginaux, vagabonds, impudents, sans futur, consommateurs de drogue, et adeptes de pratiques de sorcellerie ». 
elle offre une réalité plus complexe avec une diversité sociale, mais aussi raciale.

Lorsque l'on interroge les membres du mouvement hip-hop de Fortaleza sur les origines de leurs pratiques, bien que des variations existent quant à la version proposée, il est intéressant de constater que l'aspect profondément urbain du hip-hop, son ancrage dans certains quartiers est au cœur de leurs discours. Le South Bronx à New York revêt alors un caractère important en tant que lieu où le hip-hop serait apparu. À travers les propos de mes interlocuteurs brésiliens, se dégage des récits l'origine new-yorkaise du hiphop. Plus précisément, les acteurs pointent toujours un lien inextricable avec le ghetto, comme s'il était le référent spatial du hip-hop.

Cependant, ce rapport imagé, fantasmé à l'espace du ghetto est problématique lorsqu'il s'agit d'analyser des pratiques du hip-hop qui se déroulent de nos jours et dans un contexte «hors » Etats-Unis. En effet, au premier abord, établir un parallèle entre les Etats-Unis et le Brésil dans l'analyse du hip-hop pourrait sembler pertinent. Il est vrai que les actions menées par les organisations du mouvement hip-hop au Brésil rappellent fortement l'idéologie de la «Zulu Nation » d'Afrika Bambaataa ${ }^{4}$, un des pères fondateurs du hip-hop. De plus, en observant les pratiques du hip-hop à Fortaleza, il est aisé de se rendre compte que le hip-hop est une pratique circonscrite à l'espace de la periferia. Il serait donc tentant de résumer l'actualisation du hip-hop au Brésil à une transposition d'une pratique liée à un espace marginalisé, le ghetto, vers un autre espace qui semble souffrir des mêmes problèmes de représentation, la periferia. Dans ces deux cas, le hip-hop endosserait le rôle de représentant, de porte-parole d'une jeunesse délaissée. Cette lecture pourrait, d'ailleurs, expliquer le succès du hip-hop dans des lieux situés à une grande distance physique, mais aussi sociale, du centre irradiateur que constitue New York.

Néanmoins, cette analyse ne me satisfait pas complètement puisqu'elle n'intègre pas une perspective diachronique qui me semble fondamentale dans la compréhension du phénomène hip-hop au Brésil. Le recours à l'histoire permet de sortir d'une logique trop simpliste du type : "c'est parce qu'il y a un Brésil des favelas et des quartiers "chauds" que le hip-hop s'est vu réapproprié "logiquement" au sein des métropoles brésiliennes ».

Il s'agit dans cet article de retrouver au travers de l'histoire le processus de territorialisation du hip-hop au Brésil. Ceci nous permettra de comprendre

${ }^{4}$ C'est un DJ, Afrika Bambaataa qui a introduit la dimension sociale dans le hip-hop. Il a théorisé un cinquième élément The knowledge, qui fait partie d'une philosophie de vie que l'on retrouve dans des textes, les Infinity Lessons. Les Leçons Infinies incitent à faire preuve d'une attitude positive, à rejeter la violence et le racisme, et surtout produire le knowledge, la connaissance. Pour le Zulu, c'est uniquement par le savoir, la connaissance, que la vérité peut être atteinte ; le savoir donne la capacité à celui qui le possède de produire une pensée critique sur soi-même et la société qui l'entoure. Le pouvoir de l'esprit dans la «Zulu Nation » est donc libérateur (Mac Glyne, 2007). 
comment le hip-hop s'est fixé avec autant de force dans la periferia et comment il a été associé à une forme d'esthétique de protestation. Je reviendrai également sur ces groupements connus sous la dénomination «hip-hop organisé » au travers de mon expérience de terrain.

\section{BLACK RIO, BLACK SAMPA}

À São Paulo et à Rio de Janeiro, le tournant des années 1960/1970 est marqué par l'impulsion des musiques noires nord-américaines qui s'expriment lors des soirées dansantes (bailes) où des milliers de jeunes se rencontrent et s'amusent les fins de semaine (Dayrell, 2005). Sous l'impulsion d'équipes organisatrices de soirées ${ }^{5}$, toujours soucieuses d'apporter de la nouveauté, cette vague black permit l'introduction au Brésil d'une certaine esthétique qui inspira les personnes qui fréquentaient les bailes de façon assidue. Les projections de diapositives et de longs-métrages comme Wattstax (1973), Shaft (1971) et d'autres films classés sous la dénomination «Blaxploitation" ont permis aux danseurs soul/funk brésiliens de s'inspirer du style vestimentaire des Américains, marqué par l'influence afro. Ainsi émergèrent la mode de la coupe afro, les chaussures à talonnettes colorées, les pantalons à patte d'éléphants etc. Le style vestimentaire n'était pas le seul élément qui fut incorporé aux visuels des danseurs, ils puisaient également dans les chorégraphies de nouvelles pratiques dansantes.

Les références directes à ce qui se faisait aux Etats-Unis contribuèrent à l'expansion de ce type de soirée dédié aux musiques nord-américaines. Ce mouvement qualifié de black par la presse se transforma en un véritable phénomène de mode et eut une répercussion importante chez les jeunes des quartiers pauvres, qui voyaient dans le funk une musique qui leur appartenait (Dayrell, 2005). Les personnes qui fréquentaient ces bailes développèrent un goût prononcé pour ces éléments liés à la mode du «Black is beautiful » sans pour autant savoir que ce slogan était une référence directe à une revendication politique.

À la fin des années 1970, ce phénomène de mode prit de l'ampleur puisque sous l'impulsion du mouvement noir, s'était greffée autour des pratiques musicales toute une idéologie de lutte et de revendication identitaire. Ces militants voyaient dans la black music un instrument d'exposition d'une identité noire, mais de façon totalement positive (Dayrell, 2007).

${ }^{5}$ Ces équipes organisatrices de soirées étaient toujours soucieuses d'apporter de la nouveauté à intégrer aux bailes. Ainsi, comme le constate H. Vianna, s'est mise en place une économie autour des bailes où la reconnaissance au sein du circuit des bailes était basée sur l'acquisition des dernières nouveautés en terme de musiques, de modes et de technologie. L'exposition régulière de nouveautés garantissait en quelque sorte le succès de ces équipes organisatrices (Vianna, 1987). 
Du côté de Rio de Janeiro, la mode black s'essouffla quelque peu au début des années 1980, mais dans la cité pauliste, parce que le mouvement noir y était plus implanté, les soirées black ont perduré et joué un rôle significatif dans l'émergence du breakdance d'abord et du rap ensuite.

\section{L'IMPORTANCE DU CIRCUIT BLACK A SÃO PAULO}

Comme c'est le cas d'ailleurs dans certains pays, comme le Japon, l'Allemagne et bien d'autres, le breakdance constitua le premier élément hiphop à surgir au Brésil (Mitchell, 2002). Durant les premières années de la décennie 1980, il trouva dans ces soirées black de São Paulo, un terrain d'expression favorable.

Le breakdance a fait son apparition dans les soirées de São Paulo parce que les danseurs funk, toujours avides de nouveauté, avaient remarqué l'émergence au travers de vidéo-clips et de films, d'un nouveau style complètement différent. C'est ainsi que débarquèrent, dans les clubs de São Paulo, ces figures acrobatiques et robotiques qui tranchaient par ailleurs, avec la fluidité de la danse hustle issue du disco ${ }^{6}$ (Holman, 1984).

Le breakdance s'est implanté de façon durable à São Paulo parce que les danseurs dans l'espace des clubs pouvaient pratiquer de façon régulière et exposer leur danse, tout en agglomérant de nouvelles personnes. Ils permettaient aux $b$-boys ${ }^{7}$ de pouvoir présenter leurs pas, mais ils constituaient des lieux d'apprentissage pour les autres danseurs soucieux d'agrémenter leurs bagages techniques.

Le breakdance en tant que premier élément du hip-hop à apparaître au Brésil n'est alors aucunement une pratique porteuse d'une revendication sociale ; de plus, les jeunes des quartiers périphériques ne sont pas les seuls à pratiquer cette danse. Des témoignages, notamment ceux de Nelson Triunfo, considéré comme l'un des premiers breakers brésilien, montrent qu'une certaine dynamique est rendue possible dans le circuit local de breakdance, par les «allers-retours » de la jeunesse dorée pauliste (Rocha, Domenich, Casseano, 2001). Ces jeunes revenaient des Etats-Unis avec dans leur «bagage» des nouveaux pas qu'ils pouvaient à leur tour transmettre dans les clubs. À cette époque, la pratique du breakdance ne correspond pas, comme elle peut l'être aux Etats-Unis, à une pratique de la rue, elle est circonscrite aux night clubs à São Paulo.

Les premiers b-boys brésiliens ont fait du breakdance une danse de rue au début des années 1980 pour populariser cette danse, mais aussi parce qu'il y

${ }^{6}$ La musique disco est marquée par ses chorégraphies qui privilégient la continuité et la circularité du rythme, ce qui fait que le disco est une musique qui favorise une danse faite de mouvements fluides et sans ruptures (Holman, 1984).

7 B-boy est un synonyme de breaker. 
avait le souci de la pratiquer «à l'américaine». Ces coins de rue et places, notamment près de la station de métro São Bento, étaient des espaces où les breakers pouvaient faire des représentations en public et agréger des nouveaux danseurs pour former des groupes. Ce rythme original rencontra un fort succès, d'autant plus que les vidéo-clips de Michael Jackson et des films comme Wild Style (1983), Breakin' (1984) ou Flashdance (1983) ont accentué ce phénomène de mode. Le breakdance est allé jusqu'à apparaître dans le générique d'une novela ${ }^{8}$ en 1984. Jusque-là, le hip-hop au Brésil, se donne à voir principalement par le breakdance, sa pratique ne possède aucune dimension "sociale »; ce qui attire les danseurs, c'est son impact visuel et son esthétique différente.

Les clubs du circuit black ont permis, en parallèle de l'émergence du breakdance, l'éclosion de la première scène rap brésilienne (Dayrell, 2005). Les premiers rappeurs pouvaient s'inspirer des succès arrivant des Etats-Unis, même si le contenu passait relativement inaperçu à cause de la barrière de la langue. La musicalité particulière associant un flux de paroles sur une rythmique saccadée était appréciée (Herschmann, 2005).

La pratique du breakdance étant plus populaire, les premiers rappeurs se retrouvaient dans une situation de concurrence par rapport aux danseurs. Ils voulaient à tout prix avoir leur propre espace d'expression. Ils «abandonnèrent » de façon volontaire les lieux de rencontres des breakers afin de créer des espaces spécifiquement dédiés au rap (Rocha, Domenich, Casseano, 2001).

Cette « rupture » spatiale entre les breakers et les rappeurs de São Paulo est fondamentale car elle a rendu possible l'éclosion des premières scènes rap avec des groupes comme Stylo Selvagem, Bad Boy, Defensores do Movimento Negro, Personalidade Negra, MT Bronx, Doctor MC's et bien d'autres. En 1988 sortait le premier disque de rap brésilien, qui d'ailleurs ne fut pas un grand succès, appelé «A Ousadia do rap »".

Ce n'est qu'après la formation de la scène rap à São Paulo que l'on constate l'émergence des premiers activistes hip-hop au Brésil et l'apparition du « hiphop organisé ». Avec l'influence des rappeurs américains, les rappeurs de São Paulo transformèrent l'espace de divertissement des clubs en un espace d'affirmation de la négritude, le rap devenant un instrument porteur de lutte contre la discrimination ethnico-sociale. Le hip-hop via les rappeurs paulistes passe d'une dimension dans laquelle le ludique prédominait à des

${ }^{8}$ Une novela est un feuilleton télévisé qui passe quotidiennement au Brésil. Ces feuilletons sont extrêmement populaires au Brésil. Ces programmes à fort audimat participent également à donner le ton en matière de mode et de tendance. Participer à une novela, c'est donc acquérir la reconnaissance du grand public.

${ }^{9}$ A noter dans les noms des premiers groupes de rap paulistes l'influence des Etats-Unis (MT Bronx, Doctor MC's), du movimento negro (Defensores do Movimento Negro, Personalidade Negra) et une certaine conception du rap en tant que style associé à une esthétique agressive (Stylo Selvagem, Bad Boy). 
thématiques plus engagées, qui font écho aux situations d'extrême précarité dans la periferia pauliste.

\section{FUNK CARIOCA}

Alors qu'à São Paulo, sous l'impulsion de la vague black, le breakdance et le rap prennent place dans le quotidien de la jeunesse des quartiers périphériques paulistes, le hip-hop à Rio de Janeiro se décline de façon différente. Bien qu'étant la source de la mode black et possédant un circuit de soirées où la musique rap est très présente dans les clubs, le hip-hop s'actualisera bien différemment dans la cité carioca.

À la fin 1985, le rap est le genre musical qui sert de base aux bailes funk cariocas, $\mathrm{H}$. Vianna affirme que les programmations musicales dans les bailes sont «100\% rap». Toutefois, le terme même de hip-hop n'est pas utilisé, les funkeiros lui préfèrent le terme de funk, de balanço ou de funk pesado. Le rap remplace donc le funk en tant que musique de ces soirées, mais ce changement musical s'accompagne également d'une transformation des styles (Vianna, 1987).

Le style vestimentaire, par exemple, avait changé radicalement. Fini le visuel "afro", place au style tropical surfwear ${ }^{10}$. Il n'était plus question de mettre au centre des attentions sa couleur de peau. Le baile s'était transformé en un lieu où l'on se divertit, où l'on drague, et surtout où l'on danse. Paradoxalement, même si le breakdance bénéficia d'une certaine exposition dans les médias, la pratique corporelle du hip-hop, le breakdance ne rencontra qu'un succès limité dans la cité carioca laissant place à d'autres pratiques dansantes.

Les DJ's, quant à eux, toujours à la recherche de nouveautés, avaient trouvé dans les productions du sous-genre hip-hop appelé "Miami Bass », ce qui correspondait à leurs attentes, c'est-à-dire une musique rythmée et dansante avec une mélodie facile à retenir. Les DJ's cariocas empruntèrent la rythmique au « Miami Bass », mais également tout un décor scénique. Des chorégraphies pour le moins obscènes intègreront peu à peu les bailes funk. L'inspiration ou plutôt l'imitation est allée au bout de sa logique à partir du moment où commença la production de musiques en langue portugaise. L'apport de nouvelles technologies, spécifiquement des samplers ${ }^{11}$, joua un rôle important

${ }^{10}$ Les vêtements créés pour les surfers : des bermudas larges colorées, des tee-shirt avec des logotypes des grandes marques, les chemises à motifs tropicaux, le tout agrémenté du port de la casquette et de la paire de tennis qui fait figure de pièce importante dans la tenue du funkeiro.

${ }^{11} \mathrm{D}$ 'une façon générale, les nouvelles technologies de production musicale comme le sampling (sélectionner des échantillons dans un morceau et à le réinsérer dans une autre chanson) et le looping («à monter en boucle un échantillon donné de façon à produire un effet répétitif régulier ou aléatoire ») permettaient, sans formation connaissance musicale approfondie de 
dans la dissémination de ce style et la prolifération de nouveaux $\mathrm{MC}^{\prime}$ s, notamment du fait de la simplicité de la production.

Le hip-hop, à partir du milieu des années 1980, à Rio de Janeiro et à São Paulo est un style musical apprécié par la jeunesse de la periferia, cependant dans chacune des deux villes, le hip-hop va prendre des trajectoires distinctes. La différence entre le hip-hop de São Paulo et le hip-hop de Rio de Janeiro, devenu, funk carioca, se fait plus claire à mesure que chaque style se «brasilianise ». Les premiers activistes du mouvement hip-hop pauliste avaient d'ailleurs pris pour cible le funk carioca, en remettant en cause l'inspiration libertine et parfois violente des MC's funkeiros. Le hip-hop de São Paulo, fortement inspiré par l'influence du mouvement noir condamna le funk carioca jugé trop démoralisant et violent au contraire du rap pauliste qui se veut conscientisant.

\section{LA FORTALEZA HIP-HOP}

À la fin des années 1970 et au début des années 1980, Fortaleza comptait sur un réseau important d'équipes organisatrices qui proposaient des soirées dansantes itinérantes dans ses quartiers périphériques. Ces soirées étaient le locus de l'émergence du mouvement punk de Fortaleza et la sédimentation de la pratique du breakdance avec la formation des premiers groupes en 1984 (Damasceno, 2007). Ces soirées n'étaient pas des espaces exclusifs dédiés aux breakers, il y avait un mélange des genres, les b-boys devaient partager l'espace et donc la piste de danse avec des musicalités autres, notamment le rock et le funk. Sur le modèle des premiers breakers paulistes, les b-boys de Fortaleza avaient aussi l'habitude de faire des représentations dans les espaces publics de la ville, mais les clubs étaient les lieux d'expression privilégiés des breakers.

L'introduction au début des années 1990 du funk carioca dans les clubs de Fortaleza eut des conséquences importantes dans la pratique du breakdance à Fortaleza. Le funk carioca devint « la » musique de la jeunesse de la periferia de Fortaleza $^{12}$ et se plaça en tant que musicalité concurrente du hip-hop. Différemment de ce qui se passait à Rio de Janeiro et à São Paulo, villes dans lesquelles le hip-hop se développait distinctivement, à Fortaleza, en tant que ville réceptrice des deux influences, de façon inédite, les deux formes culturelles devaient cohabiter au sein des mêmes espaces d'expression, les clubs.

Ce qui caractérisait les soirées funk carioca à Fortaleza, c'était tout d'abord une musique particulière, inspirée nous l'avons vu du «Miami Bass », un

pouvoir produire des rythmes avec un minimum d'expérience pour peu d'être équipé d'un bon matériel (Béthune, 1999).

12 Menfis Clube, Gigantão de José Bastos, Clube do Vila União, Grémio do Ferroviário pour les plus connus. 
rythme qui ne correspondait pas aux break-beats sur lesquels les breakers pouvaient danser ${ }^{13}$. Avec une musicalité différente s'installa et se greffa également une autre façon de mobiliser la piste de danse avec, notamment le rituel du corredor, du couloir où se mettaient en scène des affrontements entre bandes rivales.

L'intérêt pour les groupes juvéniles qui fréquentaient les bailes funk résidait dans la rencontre avec un groupe rival, le plus souvent issu d'un quartier proche du sien. La cohabitation entre deux groupes « rivaux » scénarisait alors la soirée, et la rivalité entre les deux groupes de jeunes était mise en scène par le rituel du corredor.

Le baile funk dans les années 1990, que ce soit à Rio de Janeiro ou à Fortaleza, devint le lieu d'expression et surtout de visibilité des groupes juvéniles des quartiers périphériques, les galeras. Le baile funk permettait à chaque galera de se donner à voir et offrait la possibilité par le rituel du corredor d'affronter son groupe rival par des joutes corporelles violentes.

Toutefois, les galeras allaient devenir problématiques, lorsque la rhétorique violente qu'ils appliquaient dans les bailes funk, déborda dans les espaces publics et dans la vie quotidienne des citadins des grandes villes. Les affrontements ne se cantonnaient plus à l'espace de la soirée, mais pouvaient éclater dans les rues de façon spontanée par la rencontre de groupes rivaux dans un espace neutre, ou encore par le franchissement d'une "frontière " (Diógenes, 1998).

La diabolisation du funkeiro dans les médias s'intensifia au début des années 1990. Le terme de gang apparût de façon répétée pour qualifier ces groupes de jeunes. Du côté de l'action politique, fut utilisé pour répondre à ces violences urbaines le bataillon d'élite de la police militaire, le G.AT.E (Grupo de Ações Táticas Especiais). Le stigmate sur le jovem morador de periferia se renforça, d'autant plus que la différenciation entre les groupes juvéniles, les galeras et les gangs devint de plus en plus ténue dans les discours des médias.

Expulsés dans la rue par cette musicalité violente, les breakers allaient souffrir de la diabolisation du funkeiro puisque pour les Fortalezenses les breakers et les funkeiros n'étaient guère différenciables. Ils étaient associés au même univers, catégorisés sous le même profil. Ils possédaient le même vocabulaire, la même façon de s'habiller et habitaient les mêmes quartiers.

Pendant les années 1990, les hip-hoppers en tant qu'alter ego des funkeiros furent mis sur la liste des fauteurs de troubles et des éléments perturbateurs des grands centres urbains du pays. Le hip-hopper devint aux yeux des autres citadins un marginal porteur de cette esthétique dangereuse, qu'il fallait à tout prix contrôler et canaliser comme la plupart des pratiques juvéniles des jeunes de periferia.

\footnotetext{
${ }^{13}$ Les breaks-beats que les danseurs apprécient sont des parties musicales où le rythme est idéal pour pouvoir «breaker». Ainsi les Djs s'emploient à jouer des fragments de disques, la partie la plus intéressante pour les danseurs, celle où prédominaient les percussions (Toop, 1984).
} 


\section{LE HIP-HOP ORGANISÉ}

Le mouvement hip-hop de Fortaleza apparu au début des années 1990 consolida son appareil idéologique et politique contre cette stigmatisation des jeunes des periferias. Différemment du « hip-hop organizado » de São Paulo, le « hip-hop organisé » de Fortaleza n'est pas né de l'engagement de certains artistes, il ne puise pas non plus son inspiration dans le mouvement noir, mais il fut stimulé par des associations étudiantes d'extrême gauche issues des jeunesses anarchistes.

A la fin des années 1980, l'A.P, l'« Anarquia Proletária $»^{14}$ était désireuse de trouver un moyen de communiquer avec les jeunes des quartiers et de leur transmettre leur idéologie afin de les conscientiser et les politiser. L'A.P s'associa donc aux breakers pour créer le Movimento hip-hop organizado do Ceará $\left(\mathrm{MH}^{2} \mathrm{O}-\mathrm{Ce}\right)$, la première organisation du mouvement hip-hop de Fortaleza.

Le $\mathrm{MH}^{2} \mathrm{O}-\mathrm{Ce}$ réussit à unir sous sa bannière tous les groupes de danseurs de la ville. Une des premières mesures adoptées par cette organisation fut d'interdire à ses membres d'organiser les battles, c'est-à-dire des compétitions entre groupes de danseurs, qui pouvaient être interprétées comme des pratiques violentes par des non-initiés. Cette volonté de contrôler la pratique des hip-hoppers constitua une des caractéristiques du «hip-hop organisé » de Fortaleza. L'objectif étant de se différencier autant que possible de son faux frère, le funk carioca afin d'éviter l'amalgame entre ce que les acteurs appellent "os engajados $e$ os alienados $»^{15}$. Mis en concurrence avec le funk carioca, le $\mathrm{MH}^{2} \mathrm{O}-\mathrm{Ce}$ renforça son côté militant en démontrant son engagement et ses idées par une «hyperpolitisation » dans les attitudes et dans les productions musicales. Le $\mathrm{MH}^{2} \mathrm{O}-\mathrm{Ce}$ organisa et politisa les groupes de breakers et donna un ton spécifique, une orientation au style hip-hop, en termes d'influences sur les ateliers d'apprentissage des éléments hip-hop, en promouvant le rap qu'il définit comme un style $D e f^{16}$ et en censurant toutes les productions qui ne lui convenaient pas, celles assimilées au style « Miami Bass » entre autres.

\footnotetext{
14 Anarquia Proletaria est une tendance de "Movimento da Juventude" au sein du Grémio Livre Henfil du collège José Maria Campos de Oliveira du quartier de Conjunto Ceara, ce parti qui correspond aux jeunesses du PRO (Partido Revolucionário Operário), qui était un parti clandestin d'extrême gauche.

15 "Les engagés et les aliénés ». Ce terme renvoie à une brochure d'une organisation du mouvement hip-hop, témoignant de la distinction entre les hip-hoppers qui seraient militants et les funkeiros, victimes en quelque sorte de leur pratique et donnant à voir une image négative des jeunes.

${ }^{16}$ Ce style de rap se distingue par la longueur des productions musicales, il n'est pas rare que les compositions dépassent six minutes. Le contenu des chansons, les paroles du rappeur sont construits de façon plus complexe que ceux des funkeiros. La base musicale est minimaliste, la production est dépouillée, il n'y pas de place pour les mélodies dansantes au contraire du « Miami Bass », ce qui place les paroles comme élément central dans le style Def.
} 
La dénonciation d'un système, de la corruption et des violences policières devint centrale pour le mouvement hip-hop. Le hip-hop, en tant que tel, se transforma pendant ses premières années en une esthétique de la protestation. $\mathrm{Au}$ fil du temps, il se construisit un lien indéfectible entre le mouvement hiphop de Fortaleza avec sa periferia, puisque d'une certaine manière, il devint son locus principal en même temps que son représentant attitré.

Les années 2000 furent marquées par une "pulvérisation » du $\mathrm{MH}^{2} \mathrm{O}-\mathrm{Ce}$, notamment par des dissidences au sein de cette organisation, principalement en ce qui concerne son positionnement politique considéré trop à gauche. Ceci n'empêcha pas la multiplication de groupements et d'associations se plaçant dans la lignée des actions du $\mathrm{MH}^{2} \mathrm{O}-\mathrm{Ce}$. Aujourd'hui, nombreuses sont les organisations qui se revendiquent du mouvement hip-hop. On pourrait citer CUFA (Central Unica das Favelas), "Força Hip-hop », "Projeto Enxame », "Movimento Comunidade Reunida Hip-hop», "União Nordestina de Bboys » et bien d'autres. Il y a certes une pluralité dans les approches, mais $\mathrm{MH}^{2} \mathrm{O}-\mathrm{Ce}$ apparaît comme un modèle de fonctionnement pour ces organisations dans la volonté des militants à s'engager envers les jeunes des quartiers, dans les méthodes de transmission d'un " état d'esprit » hip-hop et dans l'importance que prennent les ateliers d'art hip-hop dans le fonctionnement de ces associations.

Ce qui se dégage des situations de recherche et de ma connaissance des militants du mouvement hip-hop à Fortaleza, c'est en premier lieu l'expérience de la vie dans les quartiers périphériques. Ainsi, l'appartenance à un quartier, à cet espace de la periferia, est mise en avant par les acteurs. Ces militants ont donc un rapport particulier avec leur lieu de résidence, ce qui paraît fort logique étant donné que leurs quartiers constituent également le lieu où ils ont grandi, ont construit des amitiés et tissé leur réseau de connaissances. Leur attachement pour leurs quartiers est palpable. D'ailleurs, à chaque fois que je visitais en leur compagnie leur área, ils étaient assez fiers de se montrer, mais aussi de m'exposer les bienfaits de leurs actions. Ces militants connaissent quantité de personnes dans leurs quartiers et sont très appréciés pour leur service à la comunidade. C'est en tous les cas, ce que les paroles assez sympathiques envers eux ont laissé transparaître. L'espace du quartier, du bairro, de la comunidade, constituerait, pour ainsi dire, leur monde.

Cependant, bien plus que d'être issu de la periferia, ce qui apparaît comme central dans le discours des acteurs du mouvement hip-hop, c'est leur connaissance de la rue. Être de «la rue » constituerait pour ces hip-hoppers une chose bénéfique, puisque par cette connaissance intime de la rue, de ses normes, de ses codes implicites, ils seraient dans la capacité de parler le même langage que les jeunes à qui ils s'adressent. En tout cas, ils estiment que le message, même s'il se fait autoritaire, "passe » mieux que s'il émanait d'une autorité autre comme celle des enseignants ou des forces de l'ordre. Ainsi, ils se pensent légitimes aujourd'hui pour former les jeunes aux arts hip-hop. Ils se sentent investis d'une mission de conscientisation sur les méfaits de la rue 
et de la violence. Le rapport ici à l'espace de la periferia est renversé puisque finalement chez les acteurs du mouvement hip-hop, avoir une expérience de la rue constitue un atout si elle est mise au service des jeunes du quartier.

Bien que la dure réalité de ces quartiers soit toujours mise en avant dans les entretiens que j'ai menés, les personnes qui se sont engagées dans le «hip-hop organisé " ont un point de vue assez objectif quant aux problèmes et aux plaintes des habitants de leurs quartiers. Ainsi, aucun militant ne va affirmer que tout va bien et que tout se passe pour le mieux dans sa zone d'influence. Ce qu'ils n'acceptent pas, en revanche, ce sont les caricatures et les préjugés sur les habitants des quartiers périphériques qui sont très largement véhiculés par les médias. Le fait d'avoir été soumis à un régime discriminatoire parce qu'ils venaient de la periferia est souvent souligné; dans la même ligne $\mathrm{d}^{\prime}$ analyse, les rapports conflictuels avec les policiers apparaissent centraux. Conscients des difficultés du quotidien, ces militants n'ont pas peur d'assumer leur côté periferia contrairement à ceux qui cacheraient leur lieu d'origine dans les interactions de la vie quotidienne, dans la recherche d'un emploi, par exemple. Pour ces militants, il faut avant toute chose s'assumer, être fier et montrer de quoi sont capables os jovens pobres de periferia ${ }^{17}$. Cela passe par un travail intense au niveau local, au niveau de la comunidade, du quartier, où va prendre forme l'engagement de ces militants.

Les actions des organisations du mouvement hip-hop prennent corps dans des ateliers dans lesquels, à partir des éléments du hip-hop, les jeunes vont être sensibilisés aux " problèmes » de la rue. Plus précisément tous les ateliers d'apprentissage sont émaillés de moments pendant lesquels se forment des cercles de discussions où sont abordés des sujets sensibles de la vie quotidienne dans les quartiers périphériques. Les arte-educadores, les artéducateurs, comme se définissent certains militants, soulignent souvent que l'objectif des ateliers d'apprentissage des éléments hip-hop n'est pas de former des artistes d'élite ou bien des rappeurs à succès. L'objectif premier est de conscientiser le public sur l'importance de s'engager pour son quartier et de leur redonner confiance en eux. Le plus important, c'est de resgatar a autoestima, de redonner une estime de soi à ces jeunes qui sont victimes, selon les militants, d'un manque de "lecture positive » de la part de la société, et de leur montrer qu'ils ont les capacités à réaliser des choses. Dès lors, la transmission d'un " état d'esprit », dans laquelle la discipline et le respect sont des valeurs fondamentales, est aussi une dimension importante qui apparaît dans les ateliers puisque corrélative de l'apprentissage des éléments du hiphop.

\footnotetext{
${ }^{17}$ Jeunes pauvres habitants de la periferia.
} 


\section{CONCLUSION}

Il est essentiel d'introduire la question spatiale afin de comprendre le hiphop au Brésil. De fait, si l'on prend comme étude de cas la ville de Fortaleza, on s'apercevra aisément que le hip-hop est une pratique associée à des espaces largement discriminés, les quartiers dits périphériques, la periferia.

Il est vrai que les pratiques du hip-hop ne se donnent guère à voir dans la ville de Fortaleza, si ce n'est dans les locaux des groupements du «hip-hop organisé ", qui ont leurs bases dans ces quartiers à l'aura sulfureuse. Au travers des ateliers d'apprentissage $\mathrm{du}$ hip-hop, les militants de ces associations ont pour objectif de réaliser un travail de conscientisation et de prévention auprès des jeunes habitant ces quartiers. Les organisations du mouvement hip-hop de Fortaleza sont, en un sens, ancrées dans la periferia, c'est-à-dire que les militants ne sont pas des personnes extérieures à la comunidade, ce qui leur confère une sorte de légitimité dans les actions qu'ils entreprennent pour le «vivre-ensemble » du lieu. Ce que l'on peut lire en filigrane, c'est une volonté des habitants des quartiers périphériques de trouver des solutions «eux-mêmes" à des problèmes auxquels ils sont confrontés.

À Fortaleza, il ne serait pas faux aujourd'hui de considérer le «hip-hop organisé » comme le porte-parole des quartiers «vulnérables » compte-tenu du leadership acquis par ces organisations et de leurs visibilités dans les médias locaux. Il est vrai que les activistes de ces groupements jouent aussi le rôle de leaders communautaires, et à ce titre, ils bénéficient également d'une certaine reconnaissance dans les médias ou dans les meetings politiques en tant que porte-parole ou représentant de quartiers. À un niveau supérieur, il semblerait également que le "hip-hop organisé » soit devenu un interlocuteur privilégié des pouvoirs publics. Ces derniers n'hésitant pas à considérer les organisations du mouvement hip-hop comme des «spécialistes de la periferia »; les plus à même à résoudre les "problèmes » liés à ces espaces ${ }^{18}$.

Les chercheurs travaillant sur le hip-hop ont très largement consacré le hiphop comme une forme culturelle issue des ghettos américains. Ce que j'ai avancé à propos du «hip-hop organisé » de Fortaleza pourrait en un sens conforter l'idée que le hip-hop trouverait dans les espaces marginalisés, de par le monde, un terreau favorable $d^{\prime}$ expression et d'actualisation en tant que « représentant» de ces espaces.

Néanmoins, comme j'ai essayé de le montrer avec l'histoire de l'émergence du hip-hop au Brésil, le processus qui amena le hip-hop à se territorialiser et à devenir une esthétique de la protestation est plus complexe.

Le hip-hop au Brésil a construit son identité et ses références en plusieurs phases qui sont particulières à chaque région où ce dernier s'est implanté.

18 Je reprends en substance les termes utilisés par le maire d'une ville proche de Fortaleza lors d'un entretien. 
Pour le cas de São Paulo, profitant du réseau du mouvement noir et d'une scène rap plus engagée, le hip-hop va vite devenir le locus des luttes contre les discriminations et créer par le travail des breakers un lien fort avec la rue. À Rio de Janeiro, bien que cette ville soit le lieu qui donna naissance à la vague black, le hip-hop mode carioca se cristallisa dans les mornes de la ville, produisant une esthétique particulière, pas de breakdance, mais beaucoup de rap "léger », façon sud des Etats-Unis porté par des rythmes saccadés, parfait pour le booty shaking ${ }^{19}$ et autres chorégraphies libidineuses avec par exemple a dança do сreú ${ }^{20}$.

Le hip-hop à Fortaleza ne peut se comprendre si l'on ne se saisit pas de son orientation sociale. L'engagement envers le quartier, la comunidade, que l'on retrouve chez les acteurs du hip-hop de Fortaleza ne provient d'ailleurs pas d'une application des théories de la «Zulu Nation» ou bien d'une version locale du «hip-hop organisé» pauliste. La dimension politique du mouvement hip-hop de Fortaleza est à chercher dans l'influence fondamentale du mouvement étudiant anarchiste, qui est à l'origine même de la formation idéologique des breakers et des rappeurs de Fortaleza. Le mouvement hip-hop de Fortaleza a trouvé dans la periferia un terrain adéquat quant au développement de son action politique dans laquelle la lutte contre la stigmatisation des jeunes des quartiers périphériques est importante. Aujourd'hui le hip-hop de Fortaleza se caractérise par ce lien essentiel à la periferia et par son discours hyperpolitisé, radical dans l'opposition qu'il fait entre la société et les jovens pobres moradores de periferia.

Il est important de souligner le travail important du «hip-hop organisé », il offre l'opportunité à des jeunes de se former dans une discipline artistique et d'affirmer son identité de manière plus positive. Cependant, par le rôle que le « hip-hop organisé » a acquis au cours du temps, on pourrait se poser la question du transfert de responsabilité des pouvoirs publics à des organisations du secteur associatif. Il serait intéressant d'analyser ce processus qui s'apparente à un désengagement de l'État dans la résolution des problèmes inhérents aux espaces dits "vulnérables ». De même, considérer le hip-hop comme la panacée dans la résolution des « pathologies urbaines » ne reviendrait-il pas d'une certaine manière, à réactiver le stigmate territorial sur ces populations?

${ }^{19}$ On retrouve cette danse dans certains clips de rap mettant en scène des femmes, ou plutôt les fesses de celles-ci. Les danseuses de booty shaking se caractérisent souvent par la proéminence de leurs fesses et leur capacité à les faire vibrer sans pour autant décoller les pieds du sol. La danseuse garde alors en permanence deux appuis sur le sol, compliquant le mouvement.

${ }^{20}$ Dança do creú fait partie de ces danses qui apparaissent et disparaisse au gré de la mode du moment. Ces danses qui accompagnent des chansons précises, sont mises en scène par des danseuses au nom évocateur (mulher melancia, femme-pastèque, mulher-melão, femme-melon, mulher-morango, femme-fraise) donné en référence à la taille et à la forme de leurs fesses. 


\section{BIBLIOGRAPHIE}

Hugues BAZIN, La culture hip hop, Paris, Desclée de Brouwer, 1998.

Christian BETHUNE, Le rap, une esthétique hors la loi, Paris, Éditions Autrement, 1999.

Francisco José DAMASCENO GOMES, «As cidades da juventude em Fortaleza », Revista Brasileira de História, São Paulo, vol. 37, 2007, pp. 215-242.

Juarez DAYRELL A música entra em cena, O rap e o funk na socialização da juventude, Belo Horizonte, UFMG, 2005.

Glória DIOGENES, Cartografias da cultura e da violência, São Paulo, Anablume, 1998.

Ulf HANNERZ, Explorer la ville, Eléments d'anthropologie urbaine, Paris, Les Éditions de Minuit, 1983.

Micael HERSCHMANN, O funk e o hip-hop invadem a cena, Rio de Janeiro, Universidade

Federal do Rio de Janeiro Editora, 2005.

Michael HOLMAN, "Breaking The History », in Breaking and the New York city breakers,

New York, Freudlinch Books, 1984.

Michel KOKOREFF, La force des quartiers. De la délinquance à l'engagement politique, Paris, Payot-Rivages, 2003.

Aine Mc GLYNN, « The Infinity Lessons of The Universal Zulu Nation », in Mickey HESS, Icons of hip hop: an encyclopedia of the movement, music, and culture (Volume 1), Wesport, Greenwood Press, 2007, pp. 269-270.

Tony MITCHELL, Global Noise: Rap and hip-hop outside the U.S.A, Middleton, Wesleyan

University Press, 2001.

Marcos Aurélio PAZ TELLA, "Reação ao estigma : O rap em São Paulo », Enfoques, vol.5, n¹, 2006, pp. 24-45.

Janaina ROCHA, Mirella DOMENICH, Patrícia CASSEANO, Hip hop, a periferia grita, São

Paulo, Fundação Perseu Abramo, 2001.

Tricia ROSE, Black Noise, Rap Music and Black Culture in Contemporary America, Middletown, Wesleyan University Press, 1994.

José B. da SILVA, «A cidade contemporânea no Ceará », in Simone de SOUZA (dir.), Uma nova história do Ceará, Fortaleza, Demócrito Rocha, 2000, pp. 216-236.

Livio SANSONE, Negritude sem etnicidade, o local e o global nas relações raciais e na produção cultural negra do Brasil, Salvador et Rio de Janeiro, Edufba/Pallas, 2003.

Lícia VALLADARES, "Qu’est-ce qu'une favela? », Cahier des Amériques, n³4, 2000, pp. 61-72.

Hermano VIANNA, O Baile Funk Carioca : Festas e Estilos de Vida Metropolitanos, Dissertação de Pos-Graduação en Anthropologie sociale, Université Fédérale de Rio de

Janeiro, 1987. 
RÉSUMÉ : Depuis le début des années 2000, le hip-hop au Brésil apparaît comme une culture musicale spécifique aux quartiers dits " sensibles » ou encore "vulnérables » de la périphérie des grands centres urbains. Une analyse de cette pratique musicale dans une approche comparative (avec par exemple, la France ou les Etats-Unis) nous conduit inévitablement à penser le hip-hop comme une esthétique essentiellement marginale ou subversive et spécifique à une certaine population. Toutefois cette approche mériterait d'être enrichie d'une perspective historique afin de comprendre le hip-hop brésilien, non pas comme la transposition in terra brasilis d'un modèle, mais plutôt comme une construction aux multiples références sociales et identitaires. $C^{\prime}$ est l'enjeu de cet article qui s'intéresse aux processus qui tendent à fixer le hip-hop au cœur des quartiers "périphériques » des villes brésiliennes. Le périphérique, en tant que lieu du hip-hop, revêt plus d'une construction historique et sociale que d'une transposition à l'identique d'une culture des ghettos nord-américains.

MOTS-CLÉS : Musique, hip-hop, lieu, périphérie, Brésil 